\title{
Evaluation of Pathologic Complete Response as a Surrogate for Long-Term Survival Outcomes in Triple-Negative Breast Cancer
}

Min Huang, $\mathrm{PhD}^{1}$; Joyce O'Shaughnessy, MD²; Jing Zhao, $\mathrm{PhD}^{1}$; Amin Haiderali, MBBS ${ }^{1}$; Javier Cortes, MD, PhD ${ }^{3,4}$; Scott Ramsey, MD, PhD5; Andrew Briggs, DPhil6; Vassiliki Karantza, MD¹; Gursel Aktan, MD¹; Cynthia Z. Qi, MBA7; Chenyang Gu, PhD; ; Jipan Xie, PhD; Muhan Yuan, MS7; John Cook, PhD; Michael Untch, MD ${ }^{10}$;

Peter Schmid, MD' ${ }^{11}$; and Peter A. Fasching, $\mathrm{MD}^{12}$

\section{ABSTRACT}

\begin{abstract}
Background: Pathologic complete response ( $\mathrm{PCR}$ ) is a common efficacy endpoint in neoadjuvant therapy trials for triple-negative breast cancer (TNBC). Previous studies have shown that $\mathrm{PCR}$ is strongly associated with improved long-term survival outcomes, including eventfree survival (EFS) and overall survival (OS). However, the trial-level associations between treatment effect on $\mathrm{PCR}$ and long-term survival outcomes are not well established. This study sought to evaluate these associations by incorporating more recent clinical trials in TNBC. Methods: A literature review identified published randomized controlled trials (RCTs) of neoadjuvant therapy for TNBC that reported results for both $\mathrm{PCR}$ and EFS/OS. Meta-regression models were performed to evaluate the association of treatment effect on pCR and EFS/ OS. Sensitivity analyses were conducted to assess the impact of divergent study designs. Results: Ten comparisons from $8 \mathrm{RCTs}$ ( $N=2,478$ patients) were identified from the literature review. The log (odds ratio) of $\mathrm{pCR}$ was a significant predictor of the log (hazard ratio) of EFS $(P=.003)$, with a coefficient of determination of $0.68(95 \% \mathrm{Cl}, 0.41-0.95)$. There was a weaker association between $\mathrm{PCR}$ and $\mathrm{OS}(P=.18)$, with a coefficient of determination of $0.24(95 \% \mathrm{Cl}, 0.01-0.77)$. Consistent results were found in the exploratory analysis and sensitivity analyses. Conclusions: This is the first study that has shown a trial-level association between pCR and survival outcomes in TNBC. By incorporating the most upto-date RCTs, this study showed a significant trial-level association between $\mathrm{PCR}$ and EFS. A positive association between $\mathrm{PCR}$ and $\mathrm{OS}$ was also recorded.
\end{abstract}

J Natl Compr Canc Netw 2020;18(8):1096-1104 doi: $10.6004 /$ jnccn.2020.7550

\footnotetext{
${ }^{1}$ Merck \& Co., Inc., Kenilworth, New Jersey; ${ }^{2}$ Baylor University Medical Center, Texas Oncology, and U.S. Oncology, Dallas, Texas; ${ }^{3} \mathrm{IOB}$ Institute of Oncology, Quironsalud Group, Madrid and Barcelona, Spain; ${ }^{4}$ Vall d'Hebron Institute of Oncology, Barcelona, Spain; ${ }^{5}$ Fred Hutchinson Cancer Research Center, and University of Washington, Seattle, Washington; ${ }^{6}$ London School of Hygiene and Tropical Medicine, London, United Kingdom; ${ }^{7}$ Analysis Group, Inc., Boston, Massachusetts; ${ }^{8}$ Analysis Group, Inc., Los Angeles, California; ${ }^{9}$ Complete HEOR Solutions, North Wales, Pennsylvania; ${ }^{10}$ Department of Gynecology, Helios Klinikum Berlin-Buch, Berlin, Germany; ${ }^{11}$ Barts Cancer Institute, Queen Mary University of London, London, United Kingdom; and ${ }^{12}$ Department of Gynecology and Obstetrics, Comprehensive Cancer Center

Erlangen, University Hospital Erlangen, Friedrich-Alexander University Erlangen-Nuremberg, Erlangen, Germany.
}

\section{Background}

Triple-negative breast cancer (TNBC) is a molecular subtype of breast cancer (BC) that lacks expression of the estrogen receptor, progesterone receptor, or HER2. ${ }^{1-3}$ Research has shown that TNBC accounts for $10 \%$ to $20 \%$ of all patients with $\mathrm{BC}$ and, compared with other subtypes, is associated with a more aggressive phenotype and a poorer prognosis. ${ }^{1-3}$ Neoadjuvant therapy before definitive surgery has played an increasingly important role in assessing tumor response and reducing tumor size in patients with TNBC. ${ }^{4,5}$ In trials conducted in the neoadjuvant setting, successful response to therapy is commonly gauged by the observation of pathologic complete response (pCR) on microscopic examination of surgical specimens. ${ }^{6}$

Surrogate endpoints are commonly used by regulatory agencies for accelerated approval if they can reasonably predict long-term benefits. ${ }^{7,8}$ In guidelines published by the FDA and the European Medicines Agency (EMA), pCR has been recognized as a potential surrogate endpoint to support the regulatory decision of neoadjuvant intervention in high-risk early-stage BC. ${ }^{9,10}$ Surrogate endpoints are also used by health technology assessment agencies and payers to help inform reimbursement decisions. ${ }^{11-14}$ These entities stipulate 2 criteria to establish surrogacy: (1) good correlation between the surrogate endpoint and the final outcome (individual-level association) and (2) good correlation between the treatment effect on the surrogate endpoint and the treatment effect on the final outcome (trial-level association). ${ }^{8}$ Different methodologies have been proposed to measure the strength of the correlation. ${ }^{8,12}$ In the framework proposed by Ciani et al, ${ }^{8}$ a good surrogate endpoint can be established if the estimated coefficient

See JNCCN.org for supplemental online content. 
of determination $\left(R^{2}\right)$ exceeds 0.65 . In the methodological guideline published by the German health technology assessment body, the Institute for Quality and Efficiency in Health Care (IQWiG), the correlation coefficient $(R)$ is used to rate the strength of the correlation. ${ }^{12}$ The surrogate is considered to have high correlation when the lower limit of $95 \% \mathrm{CI}$ for $R$ is $\geq 0.85$, low correlation when the upper limit of $95 \%$ CI for $R$ is $\leq 0.70$, and medium correlation in other scenarios.

Thus far, the prognostic value of pCR in the neoadjuvant setting has been established based on individual-level association across all BC subtypes, ${ }^{15-17}$ with the strongest association observed in TNBC. ${ }^{13,15,17-22}$ However, when evaluating a new treatment, one often needs to know whether the observed treatment effect on the surrogate outcome may translate into a treatment effect on the final outcome. This requires establishing trial-level association. Such trial-level associations between pCR and long-term survival outcomes (event-free survival [EFS] and overall survival [OS]) have not been established in any BC subtype in previous metaanalyses. ${ }^{13,17,23,24}$ In TNBC, the study by Cortazar et $\mathrm{al}^{13}$ is the only one that has assessed trial-level association. Their analysis was based on a subset of patients among a heterogeneous population from a limited number of randomized controlled trials (RCTs) published between 2008 and 2011 and did not find significant trial-level associations between pCR and long-term survival outcomes. ${ }^{13}$ Therefore, this study aimed to provide a recent and comprehensive evaluation of trial-level association between pCR and long-term survival outcomes (EFS and OS) in TNBC.

\section{Methods}

\section{Search Strategy and Study Selection Criteria}

MEDLINE, Embase, and the Cochrane Central Register of Controlled Trials were searched for published RCTs in English up to October 2018. Conference abstracts were also searched using the Northern Light Life Sciences Conference Abstracts database from 2016. The search strategy included keywords related to BC, neoadjuvant treatment, pCR, and survival outcomes. Studies were included in the analysis if they enrolled adult patients receiving neoadjuvant treatment of locally advanced TNBC, were RCTs, and reported results for pCR and either EFS or OS by treatment arm among patients with TNBC.

\section{Study Outcomes}

Definitions of pCR varied across the included neoadjuvant trials, with the following $3 \mathrm{pCR}$ definitions used: (1) ypT0/is ypNo, the absence of invasive cancer in the breast and axillary nodes, irrespective of ductal carcinoma in situ; (2) ypT0 ypN0, the absence of invasive cancer and in situ cancer in the breast and axillary nodes; and (3) ypT0/is, the absence of invasive cancer in the breast irrespective of ductal carcinoma in situ or nodal involvement. Among the 3 definitions, ypT0/is ypN0 and ypT0 ypN0 are both recommended by the FDA and EMA guidelines, ${ }^{9,10}$ and ypT0/is ypN0 is the most commonly used definition for TNBC studies. ${ }^{25}$ Therefore, if a study reported pCR outcomes using more than 1 definition, the pCR definition used for the primary analysis was based on the following hierarchical order: ypT0/is ypN0, ypT0 ypNo, and ypT0/is.

The long-term survival outcomes included in the analysis were EFS and OS. The EFS definition varied across the included trials and was described as EFS, disease-free survival, and relapse-free survival. For simplicity, EFS was generally used to refer to these outcomes.

\section{Statistical Methods}

\section{Association of Treatment Effects on pCR Versus on Long-Term Survival Outcomes}

The primary analysis evaluated the association of treatment effects on $p C R$ versus treatment effects on long-term survival outcomes (EFS or OS) using summary-level data for patients with TNBC from all eligible RCTs. Specifically, the treatment effect on pCR was measured using the odds ratio (OR) of the experimental versus the control arm, and the treatment effect on EFS or OS was measured using the hazard ratio (HR) for the same comparison. HRs were directly extracted from publications or estimated from digitized Kaplan-Meier curves if not reported. Each comparison between the experimental arm and the control arm contributed to 1 observation in the analysis.

Weighted linear regressions were performed with the $\log (\mathrm{HR})$ of EFS or OS as the dependent variable and the $\log (\mathrm{OR})$ of pCR as the independent variable (a natural-based logarithm was used in the study); weights were based on the number of patients with TNBC in each comparison. A negative coefficient for the $\log (\mathrm{OR})$ of pCR indicated that an increase in the OR of pCR was associated with a decrease in the HR for EFS or OS. Weighted linear regression analyses with and without a fixed intercept were performed. When the intercept was fixed, an OR of 1.0 for pCR was assumed to correspond to a survival HR of 1.0 for EFS or OS. The fixedintercept model was applied to reflect the assumption that the regression line passes through the origin corresponding to no treatment effect for both measures simultaneously.

For each model, the $R^{2}$ and its $95 \%$ CI were calculated to measure the trial-level associations between the treatment effects on pCR and EFS/OS. The 95\% CIs for 
the $R^{2}$ were estimated using the percentile method with 10,000 bootstrap iterations.

\section{Exploratory Analysis}

The surrogacy of pCR was further explored by including the TNBC data from 4 RCTs (ie, GeparTrio, ${ }^{26}$ GeparQuattro, ${ }^{27,28}$ PREPARE, ${ }^{29,30}$ and EORTC $10994^{31}$ ) in the exploratory trial-level analysis conducted by Cortazar et al. ${ }^{13}$ No TNBC-specific outcomes were reported in any of those trial publications. Data from those RCTs were therefore not identified by our literature review or included in the primary analysis. The ORs of pCR and the HRs of EFS/OS from those studies were only presented in the supplementary figures of the Cortazar et al study ${ }^{13}$ and were extracted directly from those figures to enable the exploratory analysis. To assess the accuracy of the data extraction, weighted linear regressions were first performed using data from the 4 trials only, and the results were compared against those reported by Cortazar et al. ${ }^{13}$ The same model was then fitted using the pooled data from the primary analysis and the study by Cortazar et al. ${ }^{13}$

Cross-validation and multiple sensitivity analyses were also performed to test the robustness of the results (supplemental eAppendices 1 and 2, available with this article at JNCCN.org).

\section{Surrogate Threshold Effect}

The surrogate threshold effect (STE) was defined as the minimum treatment effect on the surrogate (eg, pCR) that would be necessary to predict a statistically significant nonzero effect (eg, HR $<1$ ) on the long-term survival outcome (eg, EFS or OS). ${ }^{32}$ Per the IQWiG guideline, in situations in which the validity of a surrogate remains unclear, conclusions about true endpoints can still be made by applying the STE concept if sufficiently large effects on the surrogate have been shown. ${ }^{12}$ To draw such a conclusion, the lower confidence limit of the treatment effect on the surrogate must be larger than the STE.

All statistical analyses were performed using $\mathrm{R}$ version 3.5.1 (R Foundation for Statistical Computing). A $P$ value of .05 was used to determine statistical significance.

\section{Results}

Literature Search and Description of

Included Studies

Of 1,880 publications identified from the literature search, 8 RCTs met the eligibility criteria and were included in the analysis (Figure 1). Trial publication dates ranged from 2013 to 2018. The 8 RCTs contributed 10 comparisons of neoadjuvant interventions. Two RCTs each contributed 2 comparisons per study design: 1 trial used a $2 \times 2$ factorial design to concurrently evaluate 2 investigational agents, ${ }^{33}$ and another re-randomized nonresponsive patients after 4 cycles of initial treatment assignment to assess the efficacy of a different investigational agent. ${ }^{13,34}$ There were EFS outcomes reported for all 10 comparisons, and OS outcomes were reported for 9 comparisons.

The 8 included RCTs enrolled 2,478 patients between 2006 and 2013. Median follow-up times ranged between 36 and 56.4 months. Two trials focused on patients with TNBC, whereas the other RCTs included patients with any or broader BC subtypes (eg, HER2-negative) but reported TNBC-specific results. A variety of investigational neoadjuvant interventions were evaluated, including bevacizumab in 4 RCTs, carboplatin in 3 RCTs, and nabpaclitaxel, everolimus, and anthracycline in 1 RCT each. Different pCR definitions were used across trials, of which ypT0/is ypN0 was the most common. The characteristics of the included trials are listed in Table 1. Additional patient baseline characteristics are summarized in supplemental eTable 1.

\section{Association of Treatment Effects on pCR Versus on Long-Term Survival Outcomes}

Figure 2 shows the relationship of treatment effects on pCR versus treatment effects on EFS and OS using all 10 comparisons from the included RCTs. Results of the weighted linear regression model indicated that the $\log (\mathrm{OR})$ of pCR was a significant predictor of the $\log (\mathrm{HR})$ of EFS, with an estimated coefficient of $-0.61(P=.003)$ and an $R^{2}$ of 0.68 (95\% CI, 0.41-0.95). There was a weaker association between pCR and OS, with a coefficient of $-0.49(P=.18)$ and an $R^{2}$ of 0.24 (95\% CI, 0.01-0.77).

The pCR explained a slightly higher proportion of the variance when the intercept of the weighted linear regression model was fixed such that a pCR OR of 1.0 corresponded to an HR of 1.0, with an $R^{2}$ of 0.79 (95\% CI, 0.57-0.96) for EFS and 0.41 (95\% CI, 0.07-0.84) for OS. The coefficients were similar: $-0.51(P<.001)$ for the EFS model and $-0.46(P=.046)$ for the OS model.

\section{Exploratory Analyses}

Six comparisons from 4 RCTs were included in the triallevel analysis of patients with TNBC from Cortazar et al. ${ }^{13}$ The analysis (supplemental eFigure 1), replicating the findings in Cortazar et $\mathrm{al}^{13}{ }^{13}$ derived very similar results as what was presented in that publication.

After pooling data from the trials in the Cortazar et $\mathrm{al}^{13}$ study and those in our primary analysis, we found that the association between the $\log (\mathrm{OR})$ of $\mathrm{pCR}$ and the $\log (\mathrm{HR})$ of EFS remained significant (Figure 3). For EFS, the coefficient of the $\log (\mathrm{OR})$ of pCR was $-0.45(P=.004)$ and $R^{2}$ was $0.46(95 \% \mathrm{CI}, 0.10-0.82)$. For OS, the coefficient of the $\log (\mathrm{OR})$ of pCR was $-0.31(P=.19)$ and $R^{2}$ was $0.13(95 \% \mathrm{CI}, 0.00-0.54)$. When the intercept of the 


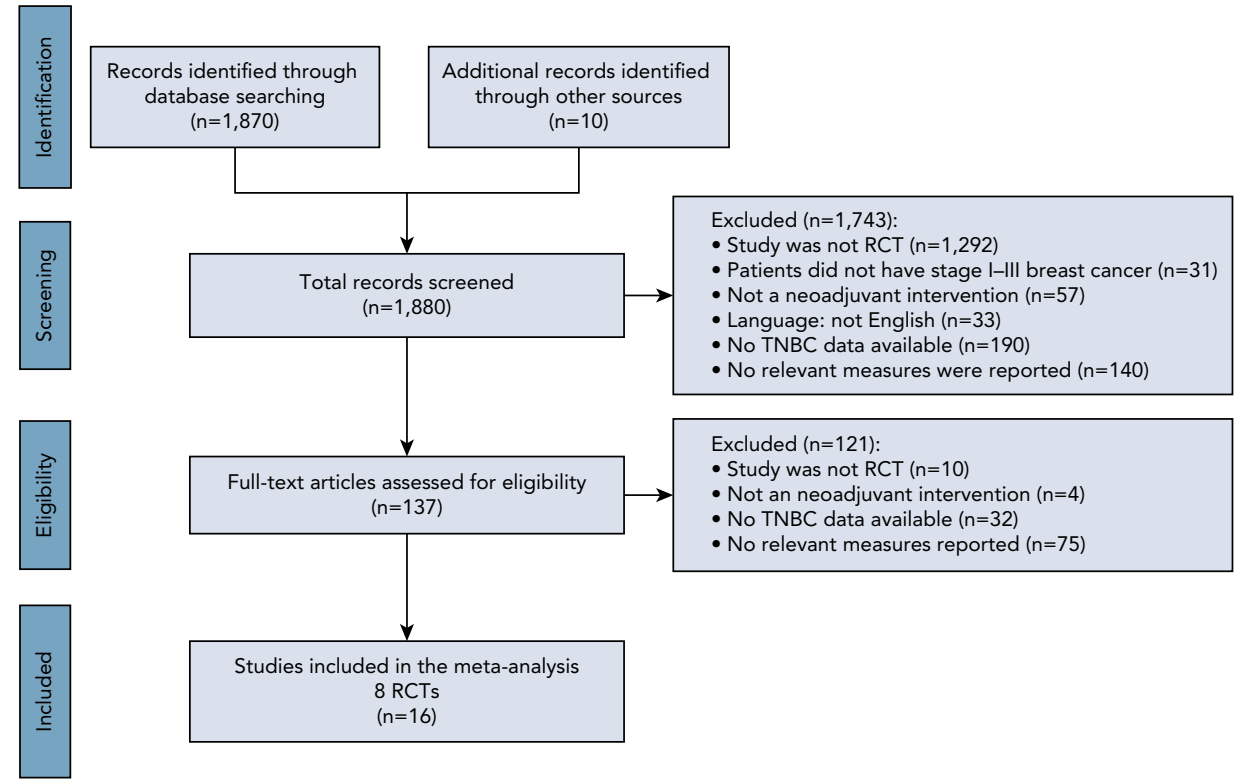

Figure 1. Study selection chart.

Abbreviations: $\mathrm{BC}$, breast cancer; $\mathrm{RCT}$, randomized controlled trial; TNBC, triple-negative breast cancer.

weighted linear regression model was fixed such that a pCR OR of 1.0 corresponded to a survival HR of 1.0 , the $R^{2}$ was 0.59 (95\% CI, 0.24-0.85) for EFS and $0.26(95 \% \mathrm{CI}$, $0.01-0.64)$ for OS.

Cross-validation confirmed the validity of the metaregression model, and sensitivity analyses results supported the robustness of the primary analysis findings (supplemental eAppendices 1 and 2).

\section{Surrogate Threshold Effect}

The STE for EFS corresponded to a pCR OR of 1.41 (Figure 2). Thus, to predict a nonzero treatment effect on EFS in a future trial with a similar treatment type, a pCR OR of at least 1.41 would need to be ascertained. No STE could be identified for OS from this analysis. Although such thresholds provide useful guidance, there will always be clinical and other judgments involved in the decision process.

\section{Discussion}

Using recently published RCTs of neoadjuvant treatment of TNBC, our study found a statistically significant association between the treatment effect on pCR and the subsequent treatment effect on EFS. With an $R^{2}$ of 0.68 for EFS in the primary analysis, pCR was a good surrogate endpoint for EFS in TNBC based on the criterion published by Ciani et $\mathrm{al}^{8}$ (ie, $R^{2}>0.65$ ). When using the IQWiG framework, we found that the association between pCR and EFS ( $R=0.82 ; 95 \% \mathrm{CI}, 0.62-0.97)$ qualified as a medium correlation. Cross-validation and sensitivity analysis results were consistent with the primary findings. Moreover, the STE for the OR of pCR in predicting a significant EFS effect was 1.41. For example, if a pCR rate was $45 \%$ in the control arm, a 9\% incremental improvement in the pCR rate would infer a significantly improved EFS for the experimental arm. These findings may serve as a foundation for future assessments of pCR as a surrogate endpoint in the neoadjuvant treatment of TNBC and can be used to assist regulatory and reimbursement decisions based on pCR.

Relative to the association between pCR and EFS, the association between pCR and OS was weaker, possibly because of the lower maturity of the OS data (note that median OS was not reached in most of the RCTs included in the analysis) and potential confounding factors, such as subsequent treatments after cancer recurrence or metastasis. This would affect the OS outcome and, in turn, the strength of the association. For example, a previous study on lung cancer showed that the association between the potential surrogate outcomes and OS is much stronger if crossover is not allowed. ${ }^{35}$ In addition, there are multiple subtypes even of TNBC, which lead to heterogeneity that could further complicate and confound this association.

To date, only a few studies have assessed the triallevel association of pCR with EFS and OS in neoadjuvant BC. Only the study conducted by Cortazar et $\mathrm{al}^{13}$ assessed the TNBC-specific trial-level association, but the analysis was exploratory and recorded little association. Our study had a specific focus on TNBC and observed a significant trial-level association between pCR and EFS in both primary and exploratory analyses. This discrepancy may be explained by the timing and design of the included trials. In the Cortazar et al study, ${ }^{13}$ all of 


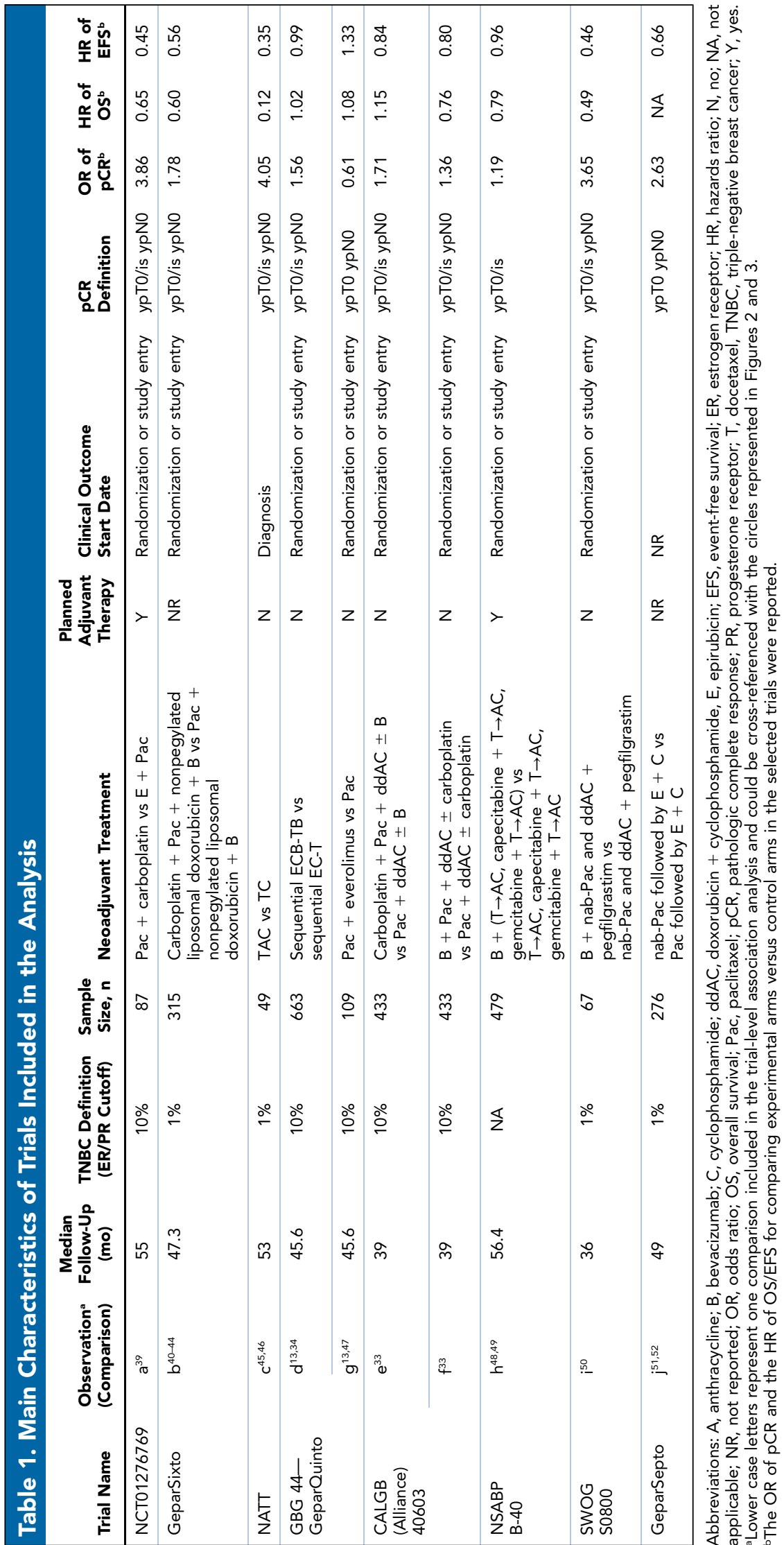


A

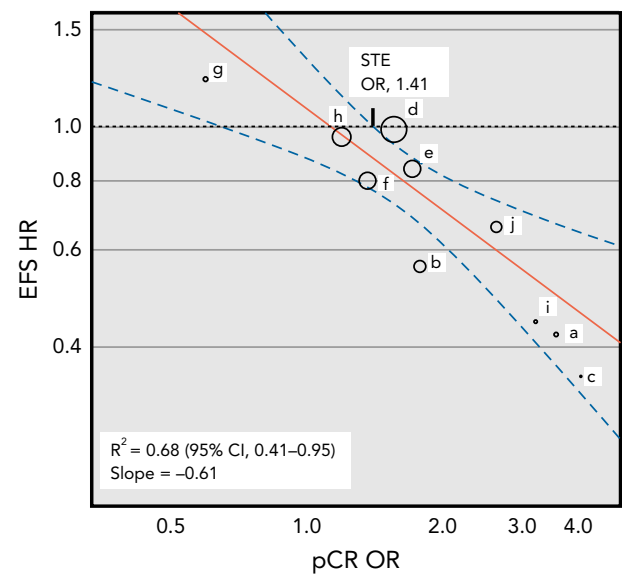

B

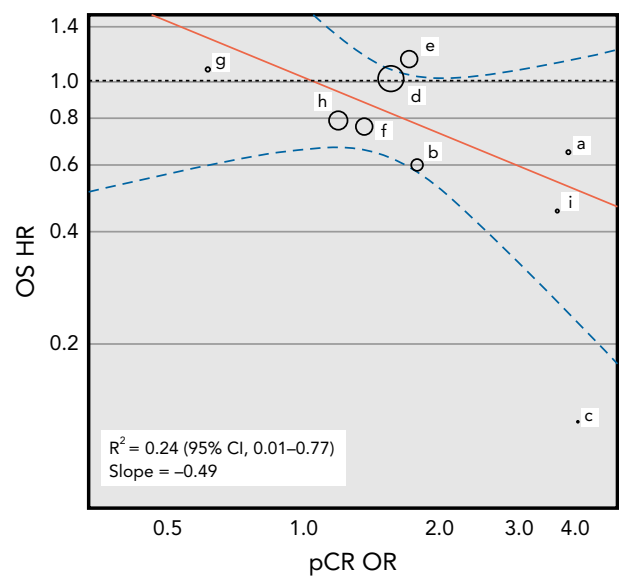

Figure 2. Trial-level association between treatment effects on $\mathrm{pCR}$ and EFS/OS based on TNBC data from trials identified in the literature search. Treatment effects are expressed as ORs for $\mathrm{PCR}$ and HRs for EFS and OS. Every circle represents a comparison of the experimental group versus the control group, with the size of the circles representing the weight of the comparison, proportional to the number of patients with TNBC in the sample. The red straight lines represent the weighted linear regression, which show the effect on (A) EFS and (B) OS predicted by the observed effects on $\mathrm{PCR}$. The blue curved lines represent the $95 \%$ prediction limits for the regression lines. The horizontal dashed lines provide a reference where HR equals 1. The STE of pCR for predicting a significant EFS effect is presented in the left panel.

Abbreviations: $E F S$, event-free survival; $H R$, hazard ratio; OR, odds ratio; OS, overall survival; $p C R$, pathologic complete response; STE, surrogate threshold effect; TNBC, triple-negative breast cancer.

the trials began before 2005 and evaluated conventional chemotherapy, whereas those included in the primary analysis of our study began after 2006 and included newer agents, such as bevacizumab, everolimus, and nab-paclitaxel. Moreover, the trials included in the
Cortazar et al study ${ }^{13}$ comprised a heterogeneous mix of BC subtypes. None of the trials in this study included TNBC as a prespecified subgroup. Therefore, those trials were not designed or powered to show treatment effects in the TNBC population and,
A

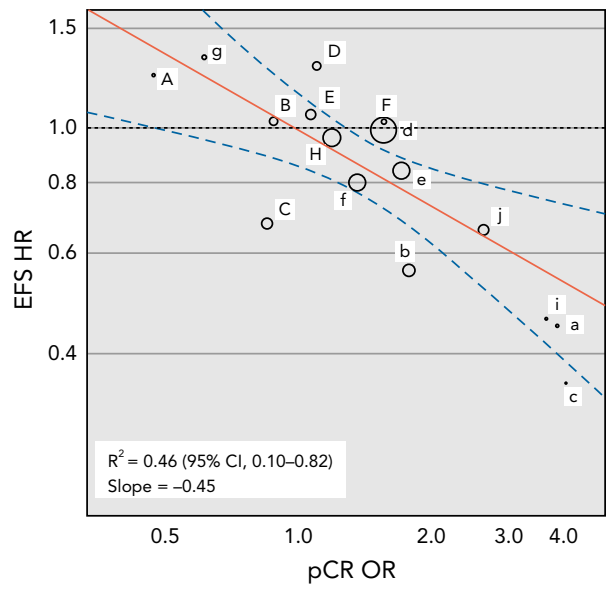

B

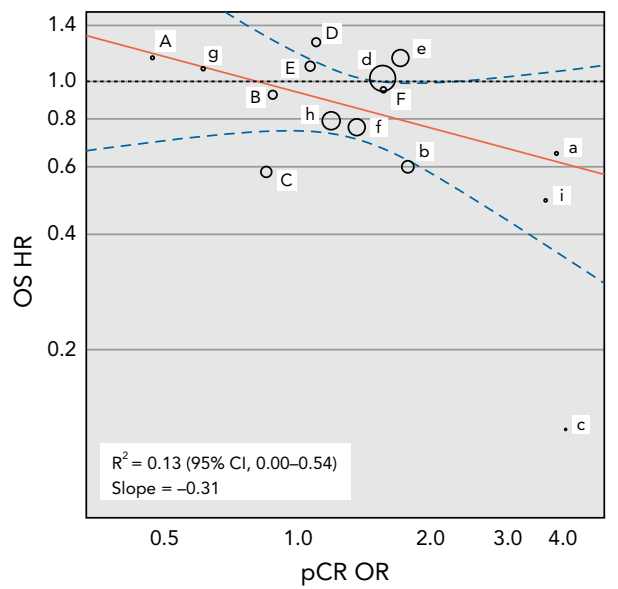

Figure 3. Trial-level association between treatment effects on $\mathrm{PCR}$ and EFS/OS based on combined TNBC data from trials identified in the literature search and Cortazar et al. ${ }^{13}$ Treatment effects are expressed as ORs for pCR and HRs for EFS and OS. Every circle represents a comparison of the experimental group versus the control group, with the size of the circles representing the weight of the comparison, proportional to the number of patients with TNBC in the sample. The red straight lines represent the weighted linear regression, which show the effect on (A) EFS and (B) OS predicted by the observed effects on PCR. The blue curved lines represent the $95 \%$ prediction limits for the regression lines. The horizontal dashed lines provide a reference where HR equals 1 . Uppercase letters represent the observations from Cortazar et al, ${ }^{13}$ and lowercase letters represent the observations identified in the literature search.

Abbreviations: EFS, event-free survival; HR, hazard ratio; OR, odds ratio; OS, overall survival; PCR, pathologic complete response; TNBC, triple-negative breast cancer. 
consequently, the trial-level associations between pCR and survival in this population were likely diluted. When considering all BC molecular subtypes, we found 4 metaanalyses that evaluated the trial-level association between pCR and long-term survival outcomes in the literature, ${ }^{13,17,23,24}$ none of which reported a significant association. The difference between the results of our study and those previous studies suggests that BC subtype and trial characteristics may be important considerations when assessing these associations. For example, Berruti et $\mathrm{a}^{23}$ suggested that the strength of trial-level association may depend on the type of treatment administered. In addition, Hatzis et $\mathrm{al}^{36}$ showed that triallevel survival benefit is a complex function influenced by many factors beyond the difference in pCR rate, and that interactions between prognostic factors and pCR may exist.

Our study is the first to focus exclusively on the triallevel association between pCR and long-term survival outcomes in TNBC. Although pCR was previously identified as a significant prognostic factor for long-term survival outcomes in TNBC based on studies assessing individual-level associations, ${ }^{13,15,16-22,25}$ no significant trial-level associations had been observed before this study. The demonstration of a trial-level association is considered the highest level of evidence (level 1) to establish surrogacy. ${ }^{8}$ Therefore, by focusing on the triallevel association, our study provides important evidence regarding the validity of pCR as a surrogate endpoint for EFS and OS in the neoadjuvant setting for TNBC.

The results of this study should be interpreted within the context of specific limitations. First, only a limited number of studies were available for analysis, which limited the precision of the estimates as evidenced by the wide CIs. Second, heterogeneity in trial design and outcome definitions may have potentially diluted the associations between pCR and long-term survival outcomes. Despite this limitation, significant associations between pCR and EFS were seen in the primary analysis and exploratory analysis including studies with divergent design and outcome definitions. Third, because of the design for 2 of the included RCTs, some patients were considered in 2 distinct comparisons, which may have affected the estimated association. However, this approach has been commonly used for surrogate validation, especially when the number of included trials was limited. ${ }^{13,17,37}$ Furthermore, although our study focused on the trial-level association, the validity of a surrogate endpoint should be supported by both trial-level and individual-level associations. Additional analyses are warranted to reassess the individuallevel association between pCR and long-term outcomes in TNBC using updated data. Finally, all TNBC neoadjuvant therapies identified from the current literature were chemotherapies. Previous studies have shown that surrogacy can vary by treatment class. ${ }^{23,38}$ Therefore, as trials investigating new agents-particularly immunotherapyare published, the association between pCR and EFS may need to be reevaluated.

\section{Conclusions}

This study builds on the existing literature by providing important insights regarding $\mathrm{pCR}$ as a surrogate endpoint for EFS and OS in the neoadjuvant treatment of TNBC. ${ }^{13}$ By combining the evidence on the trial-level association between pCR and EFS observed in this study with the individual-level association as shown by prior studies, ${ }^{13,15,16-22,25}$ pCR can be considered a reasonable surrogate endpoint for EFS in the setting of the neoadjuvant treatment of TNBC. Future research supported by a sufficient amount of data is desirable to uncover factors that may affect the validity of $\mathrm{pCR}$ as a surrogate endpoint to inform the potential impact on long-term survival outcomes of a new neoadjuvant treatment with specific trial characteristics.

Submitted December 16, 2019; accepted for publication February 18, 2020.

Author contributions: Study design, data collection, statistical analysis, data interpretation, manuscript composition and review: All authors.

Disclosures: Drs. Huang, Zhao, Haiderali, Karantza, and Aktan have disclosed that they are employed by Merck \& Co., Inc. Dr. O'Shaughnessy has disclosed that she receives personal fees from AbbVie, Agendia, Amgen AstraZeneca, Bristol-Myers Squibb, Celgene, Eisai, Genentech, Genomic Health, GRAIL, Immunomedics, Heron Therapeutics, Ipsen, Jounce, Lilly, Merck, Myriad, Novartis, Ondonate Therapeutics, Pfizer, Puma Biotechnology, Roche, Seattle Genetics, Syndax, and Nektar Therapeutics. Dr. Cortes has disclosed that he receives consultant fees from Roche, Celgene, Cellestia, AstraZeneca, Biothera Pharmaceutical, Merus, Seattle Genetics, Daiichi Sankyo, Erytech, Athenex, Polyphor, Lilly, Servier, Merck Sharp \& Dohme, GlaxoSmithKline, and Leuko; honoraria from Roche, Novartis, Celgene, Eisai, Pfizer, Samsung Bioepis, Lilly, Merck Sharp \& Dohme, and Daiichi Sankyo; grant/research support from Roche, Ariad Pharmaceuticals, AstraZeneca, Baxalta GMBH/Servier Affaires, Bayer Healthcare, Eisai, F. Hoffman-La Roche, Guardant Health, Merck Sharp \& Dohme, Pfizer, Piqur Therapeutics, Puma Biotechnology, Queen Mary University of London, and Seagen; and stock, patents, and intellectual property from MedSIR. Dr. Ramsey has disclosed that he receives consultant fees from Merck \& Co., Inc. Dr. Briggs has disclosed that he receives consultant fees from Bayer, Janssen, Novartis, Sword Health, Amgen, Merck \& Co., Inc., and Daiichi Sankyo. Ms. Qi, Dr. Gu, Dr. Xie, and Mr. Yuan have disclosed that they are employed by Analysis Group, Inc., which received consultancy fees from Merck \& Co., Inc. to conduct this study. Dr. Cook has disclosed that he received grant/research support from Merck \& Co. Inc. Dr. Untch has disclosed that he receives consultant fees and grant/research support from AbbVie, Amgen $\mathrm{GmbH}$, AstraZeneca, Celgene $\mathrm{GmbH}$, Daiichi Sankyo, Eisai GmbH, Lilly, Merck Sharp \& Dohme, Mundipharma, Myriad Genetics, Odonate, Pfizer GmbH, Puma Biotechnology, Roche Pharma AG, Sanofi Aventis Deutschland GmbH, TEVA Pharmaceuticals Ind Ltd, and Novartis; and honoraria from Bristol-Myers Squibb, Lilly Deutschland, and Pierre Fabre. Dr. Schmid has disclosed that he receives consultant fees from Pfizer, AstraZeneca, Novartis, Roche, Merck, Boehringer Ingelheim, Bayer, Eisai, Celgene, and Puma Biotechnology; and grant/research support from AstraZeneca, Genentech, Oncogenex, Novartis, and Astellas. Dr. Fasching has disclosed that he receives grant/research support from Novartis, Biontech, and Cepheid; and honoraria from Novartis, Roche, Pfizer, Celgene, Daiich Sankyo, TEVA, AstraZeneca, Merck Sharp \& Dohme, Myelo Therapeutics, Macrogenics, Eisai, and Puma Biotechnology.

Funding: This work was supported by funding from Merck (IDOE4JAG3421).

Correspondence: Min Huang, PhD, Merck \& Co., Inc., 2000 Galloping Hill Road, Kenilworth, NJ 07033. Email: min_huang@merck.com 


\section{References}

1. Plasilova ML, Hayse B, Killelea BK, et al. Features of triple-negative breast cancer: analysis of 38,813 cases from the National Cancer Database. Medicine (Baltimore) 2016;95:e4614.

2. Yao H, He G, Yan S, et al. Triple-negative breast cancer: is there a treatment on the horizon? Oncotarget 2017;8:1913-1924.

3. Gluz O, Liedtke C, Gottschalk N, et al. Triple-negative breast cancer-current status and future directions. Ann Oncol 2009;20: 1913-1927.

4. Chaudhary LN, Wilkinson KH, Kong A. Triple-negative breast cancer: who should receive neoadjuvant chemotherapy? Surg Oncol Clin N Am 2018; 27:141-153.

5. Untch M, Konecny GE, Paepke S, et al. Current and future role of neoadjuvant therapy for breast cancer. Breast 2014;23:526-537.

6. Kuroi $\mathrm{K}$, Toi M, Tsuda $\mathrm{H}$, et al. Issues in the assessment of the pathologic effect of primary systemic therapy for breast cancer. Breast Cancer 2006; 13:38-48.

7. Ciani $O$, Davis $S$, Tappenden $P$, et al. Validation of surrogate endpoints in advanced solid tumors: systematic review of statistical methods, results, and implications for policy makers. Int J Technol Assess Health Care 2014;30:312-324.

8. Ciani O, Buyse M, Drummond $M$, et al. Time to review the role of surrogate end points in health policy: state of the art and the way forward. Value Health 2017;20:487-495.

9. European Medicines Agency. The role of the pathological complete response as an endpoint in neoadjuvant breast cancer studies. Accessed April 9, 2019. Available at: https://www.ema.europa.eu/en/documents/ scientific-guideline/draft-guideline-role-pathological-complete-response-endpoint-neoadjuvant-breast-cancer-studies_en.pdf

10. United States Department of Health and Human Services. Pathological complete response in neoadjuvant treatment of high-risk early-stage breast cancer: use as an endpoint to support accelerated approval. Accessed February 21, 2020. Available at: https://www.fda.gov/ downloads/drugs/guidances/ucm305501.pdf

11. National Institute for Health and Care Excellence. Pertuzumab for the neoadjuvant treatment of HER2-positive breast cancer. Accessed April 9, 2019. Available at: https://www.nice.org.uk/guidance/ta424/documents/ final-appraisal-determination-document

12. Institute for Quality and Efficiency in Health Care: Executive Summaries. Validity of Surrogate Endpoints in Oncology: Executive Summary of Rapid Report A10-05, Version 1.1. Cologne, Germany: Institute for Quality and Efficiency in Health Care; 2005.

13. Cortazar $P$, Zhang $L$, Untch $M$, et al. Pathological complete response and long-term clinical benefit in breast cancer: the CTNeoBC pooled analysis. Lancet 2014;384:164-172.

14. United States Department of Health and Human Services. Clinical trial endpoints for the approval of non-small cell lung cancer drugs and biologics: guidance for industry. Accessed February 21, 2020. Available at: https://www.fda.gov/media/116860/download

15. Li J, Chen S, Chen C, et al. Pathological complete response as a surrogate for relapse-free survival in patients with triple negative breast cancer after neoadjuvant chemotherapy. Oncotarget 2017;8:18399-18408.

16. Esserman LJ, Berry DA, DeMichele A, et al. Pathologic complete response predicts recurrence-free survival more effectively by cancer subset: results from the I-SPY 1 TRIAL-CALGB 150007/150012, ACRIN 6657. J Clin Oncol 2012;30:3242-3249.

17. Broglio KR, Quintana M, Foster M, et al. Association of pathologic complete response to neoadjuvant therapy in HER2-positive breast cancer with long-term outcomes: a meta-analysis. JAMA Oncol 2016;2:751-760.

18. Rastogi P, Anderson SJ, Bear HD, et al. Preoperative chemotherapy: updates of National Surgical Adjuvant Breast and Bowel Project protocols B-18 and B-27. J Clin Oncol 2008;26:778-785.

19. von Minckwitz G, Kaufmann M, Kuemmel S, et al. Correlation of various pathologic complete response (pCR) definitions with long-term outcome and the prognostic value of $\mathrm{PCR}$ in various breast cancer subtypes: results from the German neoadjuvant meta-analysis [abstract]. J Clin Oncol 2011;29(Suppl):Abstract 1028.

20. Loibl S, Jackisch C, Lederer B, et al. Outcome after neoadjuvant chemotherapy in young breast cancer patients: a pooled analysis of individual patient data from eight prospectively randomized controlled trials. Breast Cancer Res Treat 2015;152:377-387.

21. Guiu S, Arnould L, Bonnetain F, et al. Pathological response and survival after neoadjuvant therapy for breast cancer: a 30-year study. Breast 2013; 22:301-308.
22. Sharma P, Kimler B, Ward C, et al. Prognosis of triple negative breast cancer patients who attain pathological complete response with neoadjuvant carboplatin/docetaxel and do not receive adjuvant anthracycline chemotherapy [abstract]. J Clin Oncol 2016; 34(Suppl):Abstract 1015. doi: 10.1200/JCO.2016.34.15_suppl.1015

23. Berruti A, Amoroso V, Gallo F, et al. Pathologic complete response as a potential surrogate for the clinical outcome in patients with breast cancer after neoadjuvant therapy: a meta-regression of 29 randomized prospective studies. J Clin Oncol 2014;32:3883-3891.

24. Korn EL, Sachs MC, McShane LM. Statistical controversies in clinical research: assessing pathologic complete response as a trial-level surrogate end point for early-stage breast cancer. Ann Oncol 2016;27:10-15.

25. Qi C, Huang M, Haiderali A, et al. Association between pathological complete responses and long-term survival outcomes among triplenegative breast cancer patients receiving neoadjuvant chemotherapy. Presented at the 24th NCCN Annual Conference; March 21-23, 2019; Orlando, Florida. Abstract HSR19-106.

26. von Minckwitz G, Kümmel S, Vogel P, et al. Neoadjuvant vinorelbinecapecitabine versus docetaxel-doxorubicin-cyclophosphamide in early nonresponsive breast cancer: phase III randomized GeparTrio trial. J Nat Cancer Inst 2008;100:542-551.

27. von Minckwitz G, Rezai M, Loibl S, et al. Capecitabine in addition to anthracycline- and taxane-based neoadjuvant treatment in patients with primary breast cancer: phase III GeparQuattro study. J Clin Oncol 2010 28:2015-2023.

28. Untch M, Rezai M, Loibl S, et al. Neoadjuvant treatment with trastuzumab in HER2-positive breast cancer: results from the GeparQuattro study. J Clin Oncol 2010;28:2024-2031.

29. Untch M, Fasching PA, Konecny GE, et al.. PREPARE trial: a randomized phase III trial comparing preoperative, dose-dense, dose-intensified chemotherapy with epirubicin, paclitaxel and CMF versus a standard-dosed epirubicin/cyclophosphamide followed by paclitaxel \pm darbepoetin alfa in primary breast cancer-results at the time of surgery. Ann Oncol 2011;22:1988-1998.

30. Untch M, von Minckwitz G, Konecny GE, et al.. PREPARE trial: a randomized phase III trial comparing preoperative, dose-dense, dose-intensified chemotherapy with epirubicin, paclitaxel, and CMF versus a standard-dosed epirubicin-cyclophosphamide followed by paclitaxel with or without darbepoetin alfa in primary breast cancer-outcome on prognosis. Ann Oncol 2011;22:1999-2006.

31. Bonnefoi H, Piccart M, Bogaerts J, et al. TP53 status for prediction of sensitivity to taxane versus non-taxane neoadjuvant chemotherapy in breast cancer (EORTC 10994/BIG 1-00): a randomised phase 3 trial. Lancet Oncol 2011;12:527-539.

32. Burzykowski T, Buyse M. Surrogate threshold effect: an alternative measure for meta-analytic surrogate endpoint validation. Pharm Stat 2006;5:173-186.

33. Sikov WM, Berry DA, Perou CM, et al. Impact of the addition of carboplatin and/or bevacizumab to neoadjuvant once-per-week paclitaxel followed by dose-dense doxorubicin and cyclophosphamide on pathologic complete response rates in stage II to III triple-negative breast cancer: CALGB 40603 (Alliance). J Clin Oncol 2015;33:13-21.

34. Gerber B, Loibl S, Eidtmann $\mathrm{H}$, et al. Neoadjuvant bevacizumab and anthracycline-taxane-based chemotherapy in 678 triple-negative primary breast cancers; results from the GeparQuinto study (GBG 44). Ann Oncol 2013;24:2978-2984

35. Hashim M, Pfeiffer BM, Bartsch R, et al. Do surrogate endpoints better correlate with overall survival in studies that did not allow for crossover or reported balanced postprogression treatments? An application in advanced non-small cell lung cancer. Value Health 2018;21:9-17.

36. Hatzis C, Symmans WF, Zhang Y, et al. Relationship between complete pathologic response to neoadjuvant chemotherapy and survival in triple-negative breast cancer. Clin Cancer Res 2016;22:26-33.

37. Saad ED, Squifflet $P$, Burzykowski T, et al. Disease-free survival as a surrogate for overall survival in patients with HER2-positive, early breast cancer in trials of adjuvant trastuzumab for up to 1 year: a systematic review and meta-analysis. Lancet Oncol 2019;20:361-370.

38. Buyse M, Sargent DJ, Grothey A, et al. Biomarkers and surrogate end points - the challenge of statistical validation. Nat Rev Clin Oncol 2010;7: 309-317.

39. Zhang $P$, Yin $Y, M o H$, et al. Better pathologic complete response and relapse-free survival after carboplatin plus paclitaxel compared with epirubicin plus paclitaxel as neoadjuvant chemotherapy for locally 
advanced triple-negative breast cancer: a randomized phase 2 trial. Oncotarget 2016;7:60647-60656.

40. von Minckwitz G, Schneeweiss A, Loibl S, et al. Neoadjuvant carboplatin in patients with triple-negative and HER2-positive early breast cancer (GeparSixto; GBG 66): a randomised phase 2 trial. Lancet Oncol 2014;15:747-756.

41. Untch M, Schneeweiss A, Salat C, et al. Long-term survival analysis of the randomized phase II trial investigating the addition of carboplatin to neoadjuvant therapy for triple-negative (TNBC) and HER2-positive early breast cancer (GeparSixto). Ann Oncol 2017;28(Suppl 5):v43-67.

42. Hahnen E, Lederer B, Hauke J, et al. Germline mutation status, pathological complete response, and disease-free survival in triple-negative breast cancer: secondary analysis of the GeparSixto randomized clinical trial. JAMA Oncol 2017;3:1378-1385

43. Loibl S, Weber KE, Timms KM, et al. Survival analysis of carboplatin added to an anthracycline/taxane-based neoadjuvant chemotherapy and HRD score as predictor of response-final results from GeparSixto. Ann Oncol 2018;29:2341-2347.

44. von Minckwitz G, Loibl S, Schneeweiss A, et al. Early survival analysis of the randomized phase II trial investigating the addition of carboplatin to neoadjuvant therapy for triple-negative and HER2-positive early breast cancer (GeparSixto). Presented at the San Antonio Breast Cancer Symposium; December 8-12, 2015; San Antonio, Texas.

45. Chen $X, Y e G$, Zhang $C$, et al. Non-anthracycline-containing docetaxel and cyclophosphamide regimen is associated with sustained worse outcome compared with docetaxel, anthracycline and cyclophosphamide in neoadjuvant treatment of triple negative and HER2-positive breast cancer patients: updated follow-up data from NATT study. Chin J Cancer Res 2016;28:561-569

46. Chen X, Ye G, Zhang C, et al. Superior outcome after neoadjuvant chemotherapy with docetaxel, anthracycline, and cyclophosphamide versus docetaxel plus cyclophosphamide: results from the NATT trial in triple negative or HER2 positive breast cancer. Breast Cancer Res Treat 2013;142:549-558.

47. Huober J, Fasching PA, Hanusch C, et al. Neoadjuvant chemotherapy with paclitaxel and everolimus in breast cancer patients with non-responsive tumours to epirubicin/cyclophosphamide $(E C) \pm$ bevacizumab-results of the randomised GeparQuinto study (GBG 44). Eur J Cancer 2013;49: 2284-2293.

48. Bear HD, Tang G, Rastogi $P$, et al. Bevacizumab added to neoadjuvant chemotherapy for breast cancer. N Engl J Med 2012;366:310-320.

49. Bear HD, Tang G, Rastogi $P$, et al. Neoadjuvant plus adjuvant bevacizumab in early breast cancer (NSABP B-40 [NRG Oncology]): secondary outcomes of a phase 3, randomised controlled trial. Lancet Oncol 2015;16:1037-1048.

50. Nahleh ZA, Barlow WE, Hayes DF, et al. SWOG S0800 (NC CDR0000636131): addition of bevacizumab to neoadjuvant nab-paclitaxel with dose-dense doxorubicin and cyclophosphamide improves pathologic complete response $(p C R)$ rates in inflammatory or locally advanced breast cancer. Breast Cancer Res Treat 2016;158: 485-495.

51. Schneeweiss A, Jackisch C, Schmatloch S, et al. Survival analysis of the prospectively randomized phase III GeparSepto trial comparing neoadjuvant chemotherapy with weekly nab-paclitaxel with solvent-based paclitaxel followed by anthracycline/cyclophosphamide for patients with early breast cancer-GBG69 [abstract]. Cancer Res 2018, 78(Suppl 4):Abstract GS3-05.

52. Loibl S, Jackisch C, Schneeweiss A, et al. Dual HER2-blockade with pertuzumab and trastuzumab in HER2-positive early breast cancer: a subanalysis of data from the randomized phase III GeparSepto trial. Ann Oncol 2017;28:497-504.

\section{See JNCCN.org for supplemental online content.}


Supplemental online content for:

\section{Evaluation of Pathologic Complete Response as a Surrogate for Long-Term Survival Outcomes in Triple-Negative Breast Cancer}

Min Huang, PhD; Joyce O'Shaughnessy, MD; Jing Zhao, PhD; Amin Haiderali, MBBS; Javier Cortes, MD, PhD; Scott Ramsey, MD, PhD; Andrew Briggs, DPhil; Vassiliki Karantza, MD; Gursel Aktan, MD; Cynthia Z. Qi, MBA; Chenyang Gu, PhD; Jipan Xie, PhD; Muhan Yuan, MS; John Cook, PhD; Michael Untch, MD;

Peter Schmid, MD; and Peter A. Fasching, MD

J Natl Compr Canc Netw 2020;18(8):1096-1104

eAppendix 1: Leave-One-Out Cross-Validation

eAppendix 2: Sensitivity Analysis

eFigure 1: Reconstructed Trial-Level Association Between Treatment Effects on pCR and EFS/OS

eFigure 2: Leave-One-Out Cross-Validation

eTable 1: Additional Trial Characteristics

eTable 2: Subgroup Analysis Results

eTable 3: Meta-Regression Analysis Results 


\section{eAppendix 1. Leave-One-Out Cross-Validation}

A leave-one-out cross-validation methodology was used to assess the prediction accuracy of the linear regression models for the hazard ratios (HRs) of event-free survival (EFS) and overall survival (OS). The regression model was refit with one observation excluded. The observed OR for pathologic complete response (pCR) from the excluded observation was then used in the corresponding regression model to predict the HRs for EFS and OS and their associated 95\% prediction intervals. This process was repeated for every observation. Consistency in the direction of the observed and predicted HR for EFS and OS (eg, both above or both below 1.0) and whether the observed HR fell within the 95\% predicted prediction interval was evaluated.

The prediction results from the cross-validation analyses are shown in (eFigure 2). The observed and predicted HRs were consistent in the direction of treatment effect on EFS for all comparisons (eg, both HRs are either above or below HR 1.0). For OS, such consistency was observed in $67 \%$ (6 of 9) of the comparisons. Additionally, the observed HRs fell within the $95 \%$ prediction intervals of the predicted HRs in $80 \%$ (8 of 10 ) of the comparisons for EFS and $78 \%$ (7 of 9) of the comparisons for OS.

\section{eAppendix 2. Sensitivity Analysis}

To evaluate the robustness of the results in the primary analysis, 2 sensitivity analyses were performed by restricting included trials to (1) randomized controlled trials (RCTs) with at least 50 patients with triple-negative breast cancer (TNBC) and (2) RCTs reporting an odds ratio (OR) based only on the ypT0/is ypN0 definition of pathologic complete response (pCR). Furthermore, meta-regression analyses were conducted to assess the impact of different trial characteristics on the associations; trial characteristics included the definition of TNBC, the observed pCR rate in the control arm, and the use of predefined adjuvant chemotherapy. The weighted linear regression model was refitted with an interaction term between the $\log (\mathrm{OR})$ of $\mathrm{pCR}$ and an indicator variable for each of the specified trial characteristics.

Analyses performed on subsets of trials yielded findings similar to those of the primary analysis (eTable 2). The $R^{2}$ values ranged from 0.60 to 0.64 for event-free survival (EFS), and from 0.11 to 0.35 for overall survival (OS). The results of the meta-regression analyses did not reveal significant impact of trial characteristics on the association between pCR and EFS. When OS was analyzed, coefficients for the interaction effect ranged from -0.98 to 0.91 , and none were statistically significant (eTable 3). 
A

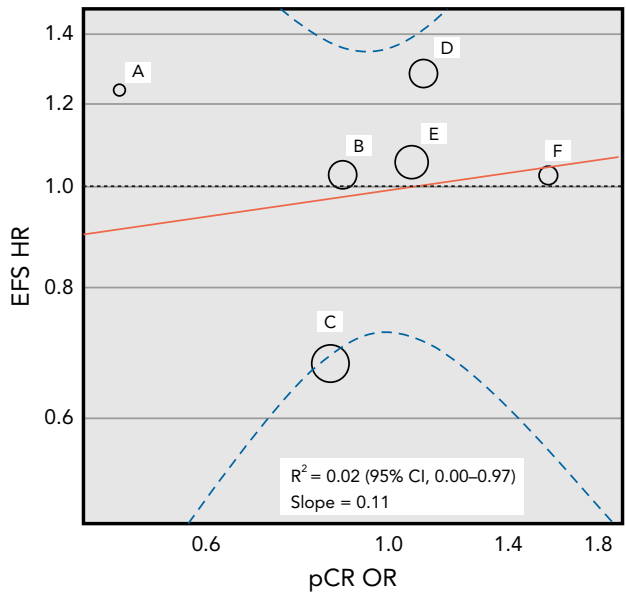

B

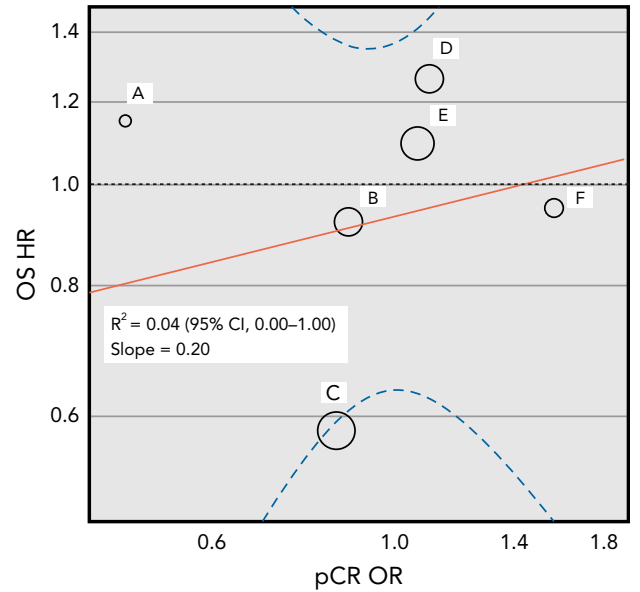

eFigure 1. Reconstructed trial-level association between treatment effects on pCR and EFS/OS based on extracted data from Cortazar et al. ${ }^{13}$ Treatment effects are expressed as ORs for $\mathrm{PCR}$ and HRs for EFS and OS. Every circle represents a comparison of the experimental group versus the control group, with the size of the circles representing the weight of the comparison, proportional to the number of patients with TNBC in the sample. The red straight line represents the weighted linear regression, which shows the effect on (A) EFS and (B) OS predicted by the observed effects on PCR. The blue curved lines represent the $95 \%$ prediction limits for the regression lines. The horizontal dashed lines provide a reference where HR equals 1.

Abbreviations: EFS, event-free survival; $H R$, hazard ratio; OR, odds ratios; OS, overall survival; $\mathrm{PCR}$, pathologic complete response; TNBC, triple-negative breast cancer.

A

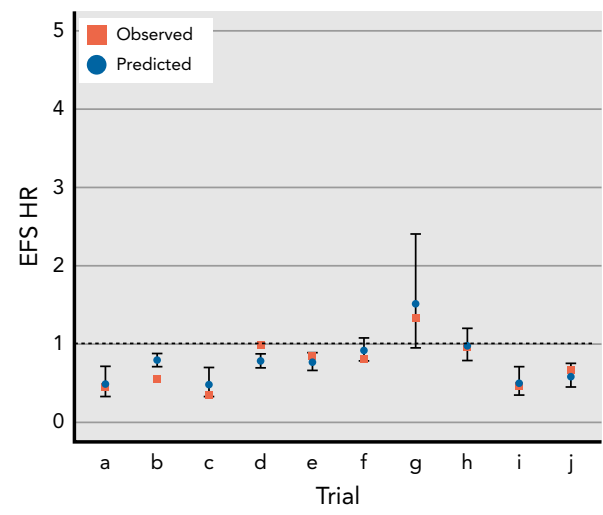

B

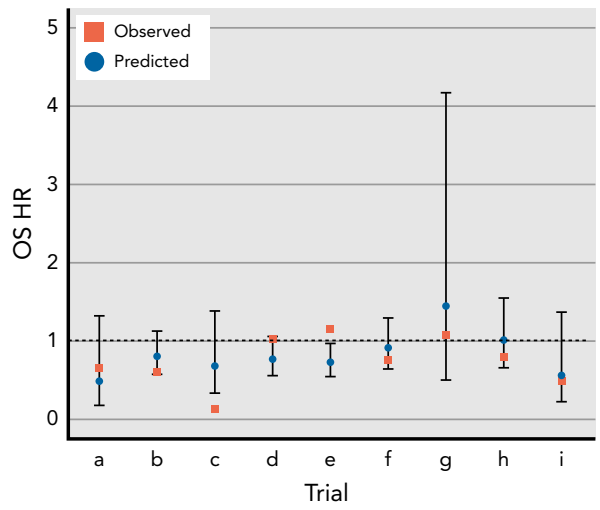

eFigure 2. Leave-one-out cross-validation. The observed HRs for (A) EFS and (B) OS for each comparison are plotted against their corresponding predicted HR and $95 \%$ prediction interval calculated from a weighted linear regression model with leave-one-out validation. Red rectangles represent the observed HR and blue circles indicate the predicted HR, with the black bars denoting the $95 \%$ prediction intervals. The horizontal dashed lines provide a reference where HR equals 1.

Abbreviations: EFS, event-free survival; HR, hazard ratio; OS, overall survival. 


\section{eTable 1. Additional Trial Characteristics ${ }^{a}$}

\begin{tabular}{|c|c|c|c|c|c|}
\hline $\begin{array}{l}\text { Trial Name/ } \\
\text { ClinicalTrials.org } \\
\text { Identifier }\end{array}$ & Neoadjuvant Treatment & pCR Rate & T Stage & N Stage & $\begin{array}{l}\text { Tumor } \\
\text { Stage/Grade }\end{array}$ \\
\hline \multirow[t]{2}{*}{ NCT01276769 } & Pac + carboplatin & $38.6 \%$ & $\begin{array}{l}\text { cT1: } 2 \% \\
\text { cT2: } 52 \% \\
\text { cT3: } 28 \% \\
\text { cT4: } 19 \%\end{array}$ & $\begin{array}{l}\text { cN0: } 28 \% \\
\text { cN1: } 28 \% \\
\text { cN2: } 36 \% \\
\text { cN3: } 9 \%\end{array}$ & $\begin{array}{l}\text { Stage II: } 34 \% \\
\text { Stage III: } 66 \%\end{array}$ \\
\hline & $\mathrm{E}+\mathrm{Pac}$ & $14.0 \%$ & $\begin{array}{l}\text { cT1: } 9 \% \\
\text { cT2: } 57 \% \\
\text { cT3: } 36 \% \\
\text { cT4: } 14 \%\end{array}$ & $\begin{array}{l}\text { cN0: } 18 \% \\
\text { cN1: } 48 \% \\
\text { cN2: } 16 \% \\
\text { cN3: } 18 \%\end{array}$ & $\begin{array}{l}\text { Stage II: } 34 \% \\
\text { Stage III: } 66 \%\end{array}$ \\
\hline \multirow[t]{2}{*}{ GeparSixto } & Carboplatin + Pac + nonpegylated liposomal doxorubicin $+B$ & $53.2 \%$ & cT3-4: 9\% & $\mathrm{cN}+: 42 \%$ & Stage III: $72 \%$ \\
\hline & Pac + nonpegylated liposomal doxorubicin $+\mathrm{B}$ & $36.9 \%$ & cT3-4: 15\% & $\mathrm{cN}+: 43 \%$ & Stage III: $78 \%$ \\
\hline \multirow[t]{2}{*}{ NATT ${ }^{\mathrm{b}}$} & TAC & $15.4 \%$ & $\begin{array}{l}\text { cT0-2: } 47 \% \\
\text { cT3-4: } 53 \%\end{array}$ & $\mathrm{cN}+: 76 \%$ & $\begin{array}{l}\text { Stage II: } 47 \% \\
\text { Stage III: } 53 \%\end{array}$ \\
\hline & TC & $4.3 \%$ & $\begin{array}{l}\text { cT0-2: } 47 \% \\
\text { cT3-4: } 53 \%\end{array}$ & $\mathrm{cN}+: 91 \%$ & $\begin{array}{l}\text { Stage II: } 33 \% \\
\text { Stage III: } 67 \%\end{array}$ \\
\hline \multirow[t]{4}{*}{$\begin{array}{l}\text { GBG } 44- \\
\text { GeparQuinto }^{\text {b }}\end{array}$} & Sequential ECB-TB & $39.3 \%$ & $\begin{array}{l}\text { cT1-3: } 88 \% \\
\text { cT4: } 12 \%\end{array}$ & $\begin{array}{l}\text { cN0: } 40 \% \\
\text { cN1: } 60 \%\end{array}$ & $\begin{array}{l}\text { Grade I: } 3 \% \\
\text { Grade II: } 53 \% \\
\text { Grade III: } 44 \%\end{array}$ \\
\hline & Sequential EC-T & $27.9 \%$ & $\begin{array}{l}\text { cT1-3: } 88 \% \\
\text { cT4: } 12 \%\end{array}$ & $\begin{array}{l}\text { cN0: } 42 \% \\
\text { cN1: } 58 \%\end{array}$ & $\begin{array}{l}\text { Grade I: } 4 \% \\
\text { Grade II: } 53 \% \\
\text { Grade III: } 43 \%\end{array}$ \\
\hline & Pac + everolimus & NR & $\begin{array}{l}\text { cT1-3: } 83 \% \\
\text { cT4: } 17 \%\end{array}$ & $\begin{array}{l}\text { cN0: } 41 \% \\
\text { cN1: } 59 \%\end{array}$ & $\begin{array}{l}\text { Grade I: } 6 \% \\
\text { Grade II: } 60 \% \\
\text { Grade III: } 34 \%\end{array}$ \\
\hline & Pac & NR & $\begin{array}{l}\text { cT1-3: } 83 \% \\
\text { cT4: } 17 \%\end{array}$ & $\begin{array}{l}\text { cN0: } 44 \% \\
\text { cN1: } 56 \%\end{array}$ & $\begin{array}{l}\text { Grade I: } 5 \% \\
\text { Grade II: } 60 \% \\
\text { Grade III: } 36 \%\end{array}$ \\
\hline \multirow[t]{4}{*}{ CALGB (Alliance) 40603} & Carboplatin $+\mathrm{Pac}+\mathrm{dd} A \mathrm{~A} \pm \mathrm{B}$ & $54.0 \%$ & $\begin{array}{l}\text { cT1: } 12 \% \\
\text { cT2: } 66 \% \\
\text { cT3: } 19 \% \\
\text { cT4: } 4 \%\end{array}$ & $\begin{array}{l}\text { cN0: } 46 \% \\
\text { cN1: } 43 \% \\
\text { cN2: } 9 \% \\
\text { cN3: } 2 \%\end{array}$ & $\begin{array}{l}\text { Stage II: } 68 \% \\
\text { Stage III: } 32 \%\end{array}$ \\
\hline & $P a c+d d A C \pm B$ & $41.0 \%$ & $\begin{array}{l}\text { cT1: } 10 \% \\
\text { cT2: } 67 \% \\
\text { cT3: } 22 \% \\
\text { cT4: } 1 \%\end{array}$ & $\begin{array}{l}\text { cN0: } 43 \% \\
\text { cN1: } 47 \% \\
\text { cN2: } 7 \% \\
\text { cN3: } 2 \%\end{array}$ & \\
\hline & $\mathrm{B}+\mathrm{Pac}+\mathrm{ddAC} \pm$ carboplatin & $52.0 \%$ & $\begin{array}{l}\text { cT1: } 13 \% \\
\text { cT2: } 64 \% \\
\text { cT3: } 21 \% \\
\text { cT4: } 2 \%\end{array}$ & $\begin{array}{l}\text { cN0: } 43 \% \\
\text { cN1: } 45 \% \\
\text { cN2: } 10 \% \\
\text { cN3: } 3 \%\end{array}$ & \\
\hline & $\mathrm{Pac}+\mathrm{ddAC} \pm$ carboplatin & $44.0 \%$ & $\begin{array}{l}\text { cT1: } 9 \% \\
\text { cT2: } 69 \% \\
\text { cT3: } 20 \% \\
\text { cT4: } 3 \%\end{array}$ & $\begin{array}{l}\text { cN0: } 46 \% \\
\text { cN1: } 44 \% \\
\text { cN2: } 8 \% \\
\text { cN3: } 2 \%\end{array}$ & \\
\hline
\end{tabular}

(continued on next page) 


\section{eTable 1. Additional Trial Characteristics ${ }^{\text {a }}$ (cont.)}

\begin{tabular}{|c|c|c|c|c|c|}
\hline $\begin{array}{l}\text { Trial Name/ } \\
\text { ClinicalTrials.org } \\
\text { Identifier }\end{array}$ & Neoadjuvant Treatment & pCR Rate & T Stage & N Stage & $\begin{array}{l}\text { Tumor } \\
\text { Stage/Grade }\end{array}$ \\
\hline \multirow[t]{2}{*}{ NSABP B- $40^{\mathrm{b}, \mathrm{c}}$} & $B+(T \rightarrow A C$, capecitabine $+T \rightarrow A C$, gemcitabine $+T \rightarrow A C)$ & $51.5 \%$ & $\begin{array}{l}\text { cT2: } 45 \% \\
\text { cT3+: } 55 \%\end{array}$ & $\mathrm{cN}+: 47 \%$ & NR \\
\hline & $T \rightarrow A C$, capecitabine $+T \rightarrow A C$, gemcitabine $+T \rightarrow A C$ & $47.1 \%$ & $\begin{array}{l}\text { cT2: } 46 \% \\
\text { cT3+: } 54 \%\end{array}$ & $\mathrm{cN}+: 47 \%$ & NR \\
\hline \multirow[t]{2}{*}{ SWOG S0800 } & $B+$ nab-Pac and ddAC + pegfilgrastim & $59.4 \%$ & NR & NR & $\begin{array}{l}\text { Stage II: } 36 \% \\
\text { Stage III: } 64 \%\end{array}$ \\
\hline & nab-Pac and ddAC + pegfilgrastim & $28.6 \%$ & NR & NR & $\begin{array}{l}\text { Stage II: } 46 \% \\
\text { Stage III: } 54 \%\end{array}$ \\
\hline \multirow[t]{2}{*}{ GeparSeptob } & nab-Pac followed by $E+C$ & $26.0 \%$ & $\begin{array}{l}\text { cT1: } 29 \% \\
\text { cT2: } 56 \% \\
\text { cT3: } 8 \% \\
\text { cT4: } 8 \%\end{array}$ & $\begin{array}{l}\text { cN0: } 65 \% \\
\text { cN1: } 33 \% \\
\text { cN2: } 2 \% \\
\text { cN3: }<1 \%\end{array}$ & NR \\
\hline & Pac followed by $E+C$ & $48.0 \%$ & $\begin{array}{l}\text { cT1: } 30 \% \\
\text { cT2: } 53 \% \\
\text { cT3: } 10 \% \\
\text { cT4: } 7 \%\end{array}$ & $\begin{array}{l}\text { cN0: } 66 \% \\
\text { cN1: } 31 \% \\
\text { cN2: } 2 \% \\
\text { cN3: } 1 \%\end{array}$ & NR \\
\hline
\end{tabular}

Abbreviations: A, anthracycline; B, bevacizumab; C, cyclophosphamide; ddAC, doxorubicin + cyclophosphamide; E, epirubicin; NR, not reported; Pac, paclitaxel; pCR, pathologic complete response; T, docetaxel; TNBC, triple-negative breast cancer.

aPatient baseline characteristics were specific to patients with TNBC if reported in the trial publications.

bData for T stage, N stage, and tumor stage/grade were based on patients with HER2-negative breast cancer or general patients with breast cancer, because the information for TNBC subpopulation was not reported.

${ }^{c} \mathrm{~T}$ stages were converted from tumor size reported. Patients with tumor size between 2 and $4 \mathrm{~cm}$ were imputed as cT2 and patients with tumors $>4 \mathrm{~cm}$ were imputed as $\mathrm{cT} 3+$.

\section{eTable 2. Subgroup Analysis Results}

\begin{tabular}{|c|c|c|c|c|}
\hline Outcome & $\begin{array}{l}\text { Number of Comparisons } \\
\text { (Observations, } n \text { ) }\end{array}$ & Coefficients for $\log (\mathrm{OR})$ & $P$ Value for Log(OR) & Model $R^{2}(95 \% \mathrm{Cl})$ \\
\hline \multicolumn{5}{|c|}{ Scenario 1: limited to comparisons with $\mathrm{pCR}$ defined as ypTO/is ypNO } \\
\hline EFS & 7 & -0.748 & .041 & $0.60(0.28-0.99)$ \\
\hline OS & 7 & -0.819 & .163 & $0.35(0.02-0.86)$ \\
\hline \multicolumn{5}{|c|}{ Scenario 2: limited to studies with a sample size of patients with TNBC $>50$} \\
\hline EFS & 9 & -0.572 & .010 & $0.64(0.32-0.96)$ \\
\hline OS & 8 & -0.237 & .413 & $0.11(0.00-0.93)$ \\
\hline
\end{tabular}

Abbreviations: EFS, event-free survival; OR, odds ratio; OS, overall survival; $\mathrm{pCR}$, pathologic complete response; TNBC, triple-negative breast cancer. 


\section{eTable 3. Meta-Regression Analysis Results}

\begin{tabular}{|c|c|c|c|c|c|c|}
\hline & Model & Coefficients & Estimate & $P$ Value & $R^{2}$ & Adjusted $R^{2}$ \\
\hline \multirow[t]{10}{*}{ EFS } & Primary analysis & $\log (\mathrm{OR})$ & -0.607 & .003 & 0.68 & 0.64 \\
\hline & \multirow{3}{*}{$\begin{array}{l}\text { Meta-regression using definition of TNBC as } \\
\text { covariate }\end{array}$} & $\log (O R)$ & -0.517 & .064 & \multirow[t]{3}{*}{0.78} & \multirow[t]{3}{*}{0.64} \\
\hline & & TNBC (ref: $\leq 10 \%$ ) & -0.457 & .271 & & \\
\hline & & Interaction term & 0.283 & .555 & & \\
\hline & \multirow{3}{*}{$\begin{array}{l}\text { Meta-regression using predefined adjuvant } \\
\text { chemotherapy as covariate }\end{array}$} & $\log (O R)$ & -0.651 & .097 & \multirow[t]{3}{*}{0.68} & \multirow[t]{3}{*}{0.52} \\
\hline & & Adjuvant (ref: Yes) & 0.000 & 1.000 & & \\
\hline & & Interaction term & 0.054 & .893 & & \\
\hline & \multirow{3}{*}{$\begin{array}{l}\text { Meta-regression using control arm pCR rate as } \\
\text { covariate }\end{array}$} & $\log (\mathrm{OR})$ & -0.591 & .032 & \multirow[t]{3}{*}{0.68} & \multirow[t]{3}{*}{0.52} \\
\hline & & Control arm pCR (ref: $\leq 30 \%$ ) & 0.041 & .900 & & \\
\hline & & Interaction term & -0.053 & .924 & & \\
\hline \multirow[t]{10}{*}{ OS } & Primary analysis & $\log (O R)$ & -0.495 & .182 & 0.24 & 0.13 \\
\hline & \multirow{3}{*}{$\begin{array}{l}\text { Meta-regression using definition of TNBC as } \\
\text { covariate }\end{array}$} & $\log (O R)$ & -0.123 & .756 & \multirow[t]{3}{*}{0.70} & \multirow[t]{3}{*}{0.47} \\
\hline & & TNBC (ref: $\leq 10 \%$ ) & 0.129 & .847 & & \\
\hline & & Interaction term & -0.976 & .285 & & \\
\hline & \multirow{3}{*}{$\begin{array}{l}\text { Meta-regression using predefined adjuvant } \\
\text { chemotherapy as covariate }\end{array}$} & $\log (\mathrm{OR})$ & -0.166 & .809 & \multirow[t]{3}{*}{0.33} & \multirow[t]{3}{*}{-0.08} \\
\hline & & Adjuvant (ref: Yes) & 0.352 & .461 & & \\
\hline & & Interaction term & -0.522 & .541 & & \\
\hline & \multirow{3}{*}{$\begin{array}{l}\text { Meta-regression using control arm pCR rate as } \\
\text { covariate }\end{array}$} & $\log (O R)$ & -0.559 & .217 & \multirow[t]{3}{*}{0.42} & \multirow[t]{3}{*}{0.06} \\
\hline & & Control arm pCR (ref: $\leq 30 \%$ ) & -0.095 & .878 & & \\
\hline & & Interaction term & 0.906 & .397 & & \\
\hline
\end{tabular}

Abbreviations: EFS, event-free survival; OR, odds ratio; OS, overall survival; pCR, pathologic complete response; TNBC, triple-negative breast cancer. 